\title{
WAKING TO NORMAL: Examining Archival ApPraisal in Data- DRIVEN SOCIETY
}

Roberta M. Fitzgerald

School of Information, College of Social and Behavior Sciences, University of Arizona

\begin{abstract}
The COVID-19 environment, where the internet is the literal lifeline and livelihood of humanity, has exposed the chasm between those equipped for technological existence and those shocked by abrupt isolation. For archives, many institutions are on an unforgiving precipice of irrelevancy. The focus of this paper is not to iterate the necessity of archival theory but to examine the position of appraisal within a technologically-driven, internet society. Of significance to this evaluation is that organization, retrieval, and use of information have evolved, and users are central players in curation cycles. Also, of importance are those archives shifting to, and innovating, decentralized digital models - and thriving. A historical overview of both fields shows that appraisal falters in technical maturation and in response to changes in how society generates, captures, and retrieves information. There exist alternate paradigms for archival roles and appraisal, however, including recognizing that users derive the interpretation of information and that transdisciplinary archivists are vital. There are also developments in digital archives where access is the bedrock of the arrangement and description and the entire appraisal process.
\end{abstract}




\section{INTRODUCTION}

"Who controls the past controls the future. Who controls the present controls the past."

- George Orwell, 1984

As we stand today, en masse, staring into the abyss of a global pandemic, George Orwell's quotation feels profoundly acute. In one jarring lurch, all society has been forcibly vaulted into digital existence, thus exposing a profound divide of those prepared for technological survival and those who are not. For archives, which exist on principals of consistency, authenticity, and integrity of information, an inherent reluctance to adopt appraisal practices for intangible records is now leaving many institutions on an unforgiving precipice of irrelevancy.

Generations of prominent archival science theorists, including Ham, Schellenberg, Kuhn, Cook, and others, have urged adapting to the management of intangible assets. But because "digital brings a variety of complexities and challenges to our archival frameworks, requiring new capabilities and approaches on how best to capture, preserve, contextualise and present the increasingly born-digital record" (Goudarouli et al, 2019), actuating change is slow. Complicating epistemological debates is the organic convergence of culture, information, technology, and sociological empowerment of the personal story. Additionally, the speed and nature of record creation and how information is accessed and shared has changed - resulting in uncertainty and confusion for information managers. James Gleick (2011) asserts, "When new information technologies alter the existing landscape, they bring disruption: new channels and new dams rerouting the flow of irrigation and transport. The balance between creators and consumers is upset: writers and readers, speakers and listeners. Market forces are confused; information can seem too cheap and too expensive at the same time. The old ways of organizing knowledge no longer work" (478). In addressing concerns about the archival community, Gauld (2018) concurs: "Put succinctly, ideas in the archival sphere are past their prime in an Age of Information" (138).

With a wealth of existing archival science papers, the focus of this paper is not to iterate the necessity of archival theory but to examine the position of appraisal within a technologically driven, internet society. Of significance to this evaluation is that organization, retrieval, and use of information have evolved, and users are central players in curation cycles. Also of importance are the archives shifting to, and innovating, decentralized digital models - and thriving. 


\section{A COLlective ARCHival BLINK}

At a base level, it is impossible to separate the story of appraisal or data sciences from the essence they share - the need to store, access, and use ever-expanding amounts of data. For archives, "The core of archival theory is about the creation, recreation and remediation of records and archives and about records being used in the course of these processes" (Klett 2019). Beginning in the late 19th century, with the publication of The Dutch Manual, archival practice establishes itself on the foundational principle of provenance and adherence to respects de fonds. Over the next 120 years, the industry exhibits contention and rhythms of epistemological reasoning that entrench analog-record theories and divert from radical advancements. Points of debate include defining records, the biases of archivists, postmodernism, and post-custodialism (Gauld 2018, Gilliland 2014, Klett 2019, Ridener 2009). Ridener (2009) explains, "The tension between appraisal and custody, between passive and active archival practice, between history "as it happened" and communication through historical records is rooted in the discourse between subjectivity and objectivity in archival theory. The tension has never been fully resolved, however, since each new paradigm also assumed many of the concepts of its predecessors. Archival theory has accepted each paradigm change cumulatively rather than successively. Today archivists continue to discuss the importance and discrepancies of both Jenkinson's and Schellenberg's theories" (143-144), theories that date from the early 1900 s to post WWll.

The digital revolution of the 1950s to 1970s reveals uncertainty in how best the archive field should readjust. Technological innovations, complexities arising from expanding organizational structures, and the introduction of intangible, digital records intimidate appraisal processes already working under a torrent of paper records. Regarding digital records, Gilliland (2014) says, "Examples of some of the issues raised by these records include blurred distinctions between drafts and record copies, multiple simultaneous originals and derivative versions of records; the generation of records in new media or new forms; and changing conceptualizations of permanence" (49). As to the industry embracing new behaviors, Ann-Sophie Klareld and Katrarina Gidlund (2017) state, "It can be difficult to reimagine the archive in the context of egovernment and the networked environment, where the complexity of archival issues increases. This is a global problem, because the requirements of digital records are different from those of paper records" (81). Today, efforts towards implementing appraisal automation and developing consensus about intangible-record management remain arduous and fragmented (Gauld 2018, Gilliland 2014, Klareld 2017, Klett 2019, Goudarouli et al, 2019).

In contrast, data science's trajectory is a narrative of steady progress and evolution. "The term "Data Science" has emerged only recently to specifically designate a new profession that expects to make sense of the vast stores of big data. But making sense of data has a long history and has been discussed by 
scientists, statisticians, librarians, computer scientists, and others for years" (Press 2013). With roots that begin before Bayes theorem in 1812, significant strides in statistics and computer technologies appear in quick succession starting in the late 1940s. With the release of Web 2.0 in the late 1990s, society's relationship with technology transitions as users create internet communities and discover unique methods of self-expression (Bell 2014, Khrystych 2019).

Between 1989 and the early 2000s, Knowledge in Databases (KKD) becomes an overarching word to describe the process of deriving meaningful information from data. KKD includes selecting data and using explicit knowledge to form logical interpretations. 'Datamining' (enacting algorithmic pattern recognition to extract data) is a piece of the KDD process. Data professionals further incorporate classification systems for search and filtration purposes, and the International Federation of Classification Societies (IFCS) recognizes 'data science' formally in 1996 (Press 2013). Press also writes that in September 2005,

$$
\begin{aligned}
& \text { The National Science Board publishes "Long-lived Digital Data Collections: Enabling } \\
& \text { Research and Education in the } 21 \text { st Century." One of the recommendations of the report } \\
& \text { reads: "The NSF, working in partnership with collection managers and the community at } \\
& \text { large, should act to develop and mature the career path for data scientists and to ensure } \\
& \text { that the research enterprise includes a sufficient number of high-quality data scientists." } \\
& \text { The report defines data scientists as "the information and computer scientists, database } \\
& \text { and software engineers and programmers, disciplinary experts, curators and expert } \\
& \text { annotators, librarians, archivists, and others, who are crucial to the successful } \\
& \text { management of a digital data collection." }
\end{aligned}
$$

By 2010 professionals and academics invite the creation of distinct specialty fields to address digital preservation, improving access, and the repurposing of data. There is a growing perception that more education in digital technology and data science is necessary for information specialists who are supporting an increasingly tech-savvy society and workforce. Scholars note that users are finding freedom and empowerment by being untethered to traditional recordkeeping practices. Technology is enabling people to retrieve and contextualize information to tell their own stories (Press 2013, Bell 2014).

Of the two, society embraces data science partly because automation and democratization of information are norms in the information age. Also, at this moment, the internet is the literal lifeline and livelihood of humanity because COVID-19 forced digital adoption over the tipping point. Gauld (2018) and Theimer (2018) argue that archival influence lags because the industry remains in a position of fear of competition 
and limits itself through circuitous debate. Archivists who persist without to change, lose the capability to participate and grow with current society.

\section{The ACTIVE Information CONSUMER}

For many theorists, understanding the interplay of information and users is key to thriving in the digital paradigm. In the twenty-first century, consumers rapidly search and filter large quantities of information at lightning speed. And each day, more data is added to the global pool of democratized information. Digital information generation is integral within societal behavior, and the digitization of analog records is a process of perpetual motion - one changes as society changes. (Carayannis et al 2015, Flinn 2007, Gauld 2018). "Technology has shifted the power away from the organization and the information professional towards the individual user. As stewards of cultural materials, archives have always managed access to, and use of, their collections, but the digital environment is radically changing cultural consumption and production patterns, obliging archives to rethink how they relate to their audiences as users of cultural content. More and more, users are expecting that the effort it takes to undertake research within an archive be lessened" (Gauld 2018, 144).

Carayannis et al (2015) writes that society now exists in a state of perpetual consumer behaviors defined as a Culture of Information - a "cultural structure composed of institutional settings and organizations that drive people to consume and produce information" (113). Rather than being passive observers, consumers are utilizing digital historical representations as dynamic objects. They are asking questions, making statements, accumulating, recombining, and repurposing digital information to reveal previously hidden context and generate new knowledge. (Gleick 2011, Carayannis et al 2015, Sternfeld 2011). We are in a "plug-in" culture, where society cannot operate if it is unplugged and where information plays a vital role in feeding/promoting certain messages and standardizing behavior" (Carayannis et al 2015, 114). Gauld (2018) advocates "developing a more symbiotic relationship with our users [so] that we shape services more to their needs and expectations; becoming increasingly flexible in our approaches to preserving records in both centralised and distributed custodial settings" (146).

By embracing widely-accepted data retrieval and semantic technologies, appraisers "can provide value added to research and policy-making by enabling mining of large amounts of information to find relationships and trends that add to the knowledge base (e.g. mining of social and mainstream media coverage of conflict areas to complement traditional sources of information). The difference between these two ends (retrieve actionable information from large amounts of knowledge and promote standardized behavior according to pre-definded messages) resides in the context and ability of the culture to transform information into knowledge" (Carayannis et al 2015, 113-114). 


\section{ACCESS Is CONTROL}

The twenty-first century is no longer about managing tangible records or providing access to consumers as a secondary thought after the appraisal process. The societal shift to selfreliance for information access is a game-changer. Gauld (2018) argues that the time is passed for archives to remain as cultural gatekeepers for analog records, especially in the twenty-first century. Continuing to force "users to adhere to policy when they have broken away from the structures and norms that such policy was designed to codify is difficult and may be counterproductive. The web as a platform empowers users and breaks both the relationship enshrined in the document metaphor between information, records and computing platform, and a user's inclination to conform" (Bell 2014, 234-235). Therefore, "in an era of digital recordkeeping, of digital curation, with its focus on reuse, multiversioning and asset management, of mass digitization and cloud storage, the central question underlying appraisal has shifted from how we appraise digital materials to should we appraise digital materials?" (Gilliland 2014, 32). Goudarouli et al (2019) encourage loosening control of record interpretation and merging access into the appraisal process. Giving access and the user priority provides support for the centricity of the information consumer. It also builds trust in archival transparency and accountability.

Many archives, however, approach digital management and access as tool-based solutions - complicating processes and avoiding reconciliation with evolving society. Bell (2014) proposes that
"technological change is doing something more vital to recordkeeping than forcing an evaluation of whether our tools and principles remain fit for purpose? Perhaps, instead, it is forcing the recordkeeping profession through the lookingglass, making it engage with some of the difficult and complex semantic and intellectual debate that has been taking place for years, but that, practically, it was possible to ignore and 'get on with things' and continue with the worthwhile and everyday work of processing and providing access to records (232).

"A consistent and compelling motivation for archivists has been the ever-increasing abundance of documentation and the resulting fear of being overwhelmed by the management of an increasingly voluminous, multimedia and documentarily complex, yet qualitatively diluted, record" (Gilliland 2014, 51). Agostinho et al (2019), however, point to the fact that appraisers are familiar with communing among disorder, and that is possible to view digital archives then as simply "the latest installment in a long negotiation ... between control and uncertainty, order and chaos, and ultimately between power and knowledge. (423). 
Institutions, like the Portuguese National Archive, Torre do Tombo, are progressing with data science integration to reduce chaos and bring methods of search and retrieval in line with contemporary internet user experiences. Doucet et al (2019) say the approach for the National Archive of Torre do Tombo is to address 'data models and description vocabularies' and also develop support for 'a graph database and an Object Graph Mapping library.' Working in conjunction with the International Committee for documentation CIDOC, which is a part of the International Council of Museums (ICOM, the goal is to create a CIDOC-CRM ontology model for cultural works of art functional for archiving purposes.

The overall goal is to build a CIDOC-CRM-compliant system for document description and retrieval, to be used by professionals and the public. The early stages described here include the design of the core data model for archival records represented as the ArchOnto ontology and its embodiment in the ArchGraph knowledge graph. The goal of a semantic archival information system will be pursued in the migration of existing records to the richer representation and the development of applications supported on the graph (168).

The user interfaces have to satisfy the diverse requirements of applications and end users. For applications, the graph must provide CRUD operations, as well as the display of subgraphs. Users can be professionals (archivists creating new records), or other users and all need to access the information on the records in an intuitive manner, while traversing the graph based on archival contents and relationships. (177)

The National Archives UK is also working to put the organization "in a position of being able to contextualise and explore the government record of today against the backdrop of over a thousand years of history interwoven with an evolving governmental and legal system" (Goudarouli et al 2019, 179). Rather than "trying to cleanse and standardise data, our techniques aim at leveraging uncertainty by quantifying and working around the 'fuzziness' found in our large-scale collections. Our aim is to enable the user to make robust, data-driven access decisions" (178).

Although "the goals of appraisal have expanded since the 1970s to include ensuring that there is adequate and systematic documentation of societal experiences and phenomena" the 1980s show electronic records archivists preoccupied with digitizing and selecting born-digital records "deemed most valuable for longterm retention" in digital recordkeeping systems subjects to "complex, networked and transjurisdictional bureaucracies" (Gillliland 2014, 32). Critics argue that using appraisal "to reduce the bulk of born-digital records" is unstainable and out of touch with users and contemporary information creation practices (32). 


\section{Transdisciplinary ApPraisers, Microcosms, ANd Macrocosms}

Data science professionals are often transdisciplinary and able to bring information management and technical skills to their professions. Theimer (2018) argues that archivists are hindered without similar training. "We need to become, if we are not already, masters of data, or to use a less romantic title, data scientists. We need to understand how to apply the tools and techniques of 'big data' to data, both big and small, that we generate in the course of our work and that is a part of our holdings...we need to know what is possible and useful so that we can work with others to generate meaningful outputs" (9). A contemporary archival role is "collaborating with citizen archivists, passionate amateurs and communities of enthusiasts, official community archives and organizational archives, whoever, to help ensure preservation of valuable records, make them accessible and promote their use" (14). Bell's (2014) is one where record making agencies are "influencing, providing perspective and helping to articulate the role of the recordkeeper and recordkeeping" (231), noting that the operations within and between agencies mixed with autonomous user behavior are obstructing traditional appraisal processes.

With evidence from findings by the United Kingdom National Archives, Gauld (2019) states that "archives are having to fight tooth-and-nail to remain viable" (145) under deteriorating financial conditions. Yet some players in the archival industry, those built on data science principles, are thriving and gaining support from the technology, philanthropy, science, and other sectors, they release information for public consumption. Organizations like the

Internet Archive, Community Heritage and Archive Group, Global Forest Watch, and the Johns Hopkins COVID-19 Dashboard are collecting, archiving, and disseminating information in realtime.

Community archives, which have skirted traditional archival methodologies and principles for decades, are also energized by opportunities afforded to them by the internet. Flinn (2007) explains that $t$ communities stimulate the global increase in the number of participatory archives due to fear of "losing their identity or having that identity marginalised or ignored" but also by digital availability.

First, there has been the impact of new technologies on both the formation of communities and on the processes of community histories or community archives. As previously indicated the development of the World Wide Web and the Internet have meant that formation of communities of shared interest (be it occupation, locality, cultural, diasporic or other) no longer need to have a physical 'meeting place' but often come together online in a virtual environment, frequently bringing together people from very diverse and widely distributed geographic locations. (158) 
These digital archive communities view the internet as being effective and convenient for storing and sharing "community memories and archives, notably of photographs, scanned documents, and...oral testimony" (Flinn 2007, 159). The Mapping a Colony website exemplifies o community formed across geographical disconnect regions. The "project engages with digital cultural heritage on a prototype website that tests the interactive potential of what we might call digital colonial fragments. It is also a project that maps places, people, and photographic material relating to Danish colonial heritage in two geographic areas: Denmark and the former Danish West Indies (now the US Virgin Islands, USVI) (blogit.itu.dk, May 10, 2020).

There are also many active initiatives in big data archives working to reframe appraisal and democratize information within their legal boundaries. Presentations from the 2019 Digital Libraries for Open Knowledge conference (Doucet et al, 2019) illustrate the breadth of solutions currently in production or in research stages. For instance, the German Broadcasting Archive (DRA), which maintains the "historical video collection of the former German Democratic Republic (GDR is implementing autoindexing so consumers can conduct searches with the aid of classification systems. "Automatic indexing is an important prerequisite to enable semantic search...In particular, visual concept classification and person recognition play an essential role to provide fine-grained access to large image and video databases" (198).

In a second example, experimental research is advancing to improve search results of historical art archives using titles using Name Entity Recognition (NER). The team shows that existing NER systems are inefficient because they "are typically limited to a few common named entity types, such as person, location, and organization" (192). The solution is to introduce semisupervised machine training (using some labeled data with large amounts of unlabeled data) "to create high-quality training data by leveraging existing cultural heritage resources." The evaluation improved "NER performance for artwork titles" (192). There are also experiments in progress to help archives select and capture web documentation.

In a third example, The Memento Tracer Framework's function "is inspired by existing capture approaches yet aims for a new balance between archiving at scale and quality of the resulting snapshots. The framework was developed as part of a project that focuses on capturing scholarly artifacts from productivity portals such as GitHub, Slideshare, Publons, Figshare, Wikipedia, and Stack Overflow" The system "puts the curator in charge of determining the desired components of a to be archived web resource and takes advantage of frequently reused patterns in online productivity portals." After a 
resource is captured, curators "can share it with the community via a publicly accessible repository, thereby crowdsourcing the web curator task." (312).

In their publication Rethinking Archives as Digital: The Consequences of "Paper Minds" in Illustrations and Definitions, Ann-Sophie Klareld and Katrarina Gidlund (2017) the impact of analog records management is having on digital management of e-governmental records. They find "Paradoxes exist: firstly, between the slow-moving and fixed constructions of legal principles; secondly, in the story of a holistic, proactive approach that is supportive of egovernment; and thirdly, between the general and shared story on the surface and the differences underneath, including different understandings of what a digital archive is, could be, or should be" (106). Regarding how this impacts society and the need for public access to the data, the authors suggest stepping back from implementing individual digital toolbased solutions to view the process more objectively. (106)

\begin{abstract}
A possible conclusion is that public agencies would benefit from taking a more holistic, proactive, better-informed view of archival systems, rather than implementing technical solutions to fulfill certain predefined purposes that assume obsolete procedures developed in the paper administration. It will otherwise be difficult to meet the demands of e-government, including co-operation among different agencies. The overall result indicates the importance of recognizing that this demand for a shared definition should be directed toward a shared clarification regarding principles if we want to create and support innovative and contextdependent value for society. It is crucial to focus on the principles to which public archives should adhere, rather than on existing practices, since the preferred result might not be supported by current normative routines (106).
\end{abstract}

Agostinho et al (2019) argue that there are advantages to integrating data science into archives. "Digital environments repurpose and refashion the logic of the archive under conditions of social, political, ecological, and technological uncertainty" (423). And by decentralizing the archival process, Ann-Sophie Klareld and Katrarina Gidlund (2017) suggests the big data "earchive will become more integrated in the business, the existing practice - wherein the archive is a separate entity or organization - still shines through" (104).

\title{
CONCLUSION
}

Modern society embraces information management and knowledge-equity through data science but also seems to be circumventing out-of-date appraisal practices. Gauld (2019 takes this a step further by 
suggesting "that the demise of ideas in an archival context is not occurring in isolation. There is a wider shift taking place whereby ideas in general society are, today, quite simply not what they used to be" (143). Carayannis et al (2015) argue that despite the challenges presented with access to magnitudes of unfettered data,

The benefits afforded by the availability and quality of key information are well recognized. For example, the ability to connect to information sources created an opportunity to obtain information at an unprecedented rate and scope, providing new platforms that equipped individuals with new ways to accumulate, obtain, and use information. As "value" is hidden in diverse and large amounts of internal and external information, and as a result finding the useful information also implies a search process, which is extended at the margins of "the known" environment with the aim of identifying emerging issues and events within a certain context (97-98).

Carayannis et al (2015) further explain that there now exists a cyclical relationship between machinedriven analytics and consumer "decision-making" (198). History shows this is a relationship that data science advocates work to advance but also that appraisal has yet to embrace technical maturation and changes in how society generates, captures, and retrieves information.

Many theorists recognize alternate paradigms for appraisal methodologies and the role of archivists. Capitalizing on the potential, however, means advocating for users as interpreters of information and for interdisciplinary training amongst archivists. Developments in digital environments also illustrate that access is the bedrock of arrangement and description and the entire appraisal process. History shows that appraising digital records requires more than administering a package of tools to manipulate intangible records. - it's about sorting and navigating society's thought processes. 


\section{REFERENCES}

Mapping a Colony 2017. Mapping a Colony. https://blogit.itu.dk/mappingacolonyen/.

Agostinho, D., A. Ring, K. Veel, C. D’Ignazio, and N. B. Thylstrup. 2019. "Uncertain Archives: Approaching the Unknowns, Errors, and Vulnerabilities of Big Data through Cultural Theories of the Archive." Surveillance \& Society 17 (3/4): 422-441.

Bell, A. 2014. "Participation Vs Principle: Does Technological Change Marginalize Recordkeeping Theory?" In Archives and Recordkeeping : Theory into Practice, edited by C. Brown, 227-252. London: Facet Publishing.

Carayannis, E. G., D. Popescu, and A. Pirzadeh. 2015. "“The Culture of Information and the Information of Culture" ." In "Arts, Research, Innovation and Society", edited by G. Bast,

E. G. Carayannis and D. Campbell, 92-124. Switzerland: Springer International Publishing.

Doucet, A. 2019. "Digital Libraries for Open Knowledge: 23rd International Conference on Theory and Practice of Digital Libraries, Tpdl 2019, Oslo, Norway, September 9-12, 2019, Proceedings."Springer, September 9-12, 2019.

Flinn, Dr Andrew. 2007. "Community Histories, Community Archives: Some Opportunities and Challenges." Journal of the Society of Archivists 28 (2) (October 1,): 151-176. doi:10.1080/00379810701611936.

Gauld, C. 2018. "The End of Archival Ideas?" In Archival Futures, edited by C. Brown, 137156. London: Facet Publishing.

Gilliland, A. 2014. "Archival Appraisal: Practising on Shifting Sands." In Archives and Recordkeeping: Theory into Practice, edited by C. Brown, 31-62. London: Facet Publishing.

Gleick, J. 2011. The Information. United States: Pantheon Books.

Goudarouli, Eirini, Anna Sexton, and John Sheridan. 2019. "The Challenge of the Digital and the Future Archive: Through the Lens of the National Archives UK." Philosophy \& Technology 32 (1).

Khrystych, Zakhar. "Data Science Timeline." Medium., last modified -08-23T05:34:08.328Z, accessed May 8, 2020, https://medium.com/@ zakharkhrystych/data-science-timeline305ef75dceb6.

Klareld, A-S and K. L. Gidlund. 2017. "Rethinking Archives as Digital: The Consequences of "paper Minds" in Illustrations and Definitions of E-Archives." Archivaria 83 (Spring): 81-108.

Klett, E. 2019. "Theory, Regulation and Practice in Swedish Digital Records Appraisal." Records Management Journal 29 (1/2) (11 March): 86-102.

Press, Gil. "A very Short History of Data Science." Forbes., last modified May 28, accessed May 7, 2020, https://www.forbes.com/sites/gilpress/2013/05/28/a-very-short-history-of-datascience/.

Ridener, John. 2009. From Polders to Postmodernism : A Concise History of Archival Theory. Duluth, MN: Litwin Books, LLC. 
Sternfeld, J. 2011. "Archival Theory and Digital Historiography: Selection, Search, and Metadata as Archival Processes for Assessing Historical Contextualization." Th American Archivist 74 (Fall/Winter). https://americanarchivist.org/doi/pdf/10.17723/aarc.74.2.644851p6gmg432h0.

Theimer, K. 2018. "It's the End of the Archival Profession as we Know it, and I Feel Fine." In Archival Futures, edited by C. Brown, 1-17. London: Facet Publishing. 